\title{
Im Diskurs bestehen. Über den notwendigen Pluralismus in der ökonomischen Politikberatung: Kommentar zum Beitrag von Sebastian Dullien und Gustav Horn
}

\author{
Stefan Kooths
}

Online publiziert: 22. Februar 2019

(C) List-Gesellschaft e.V. 2019

Die Autoren beklagen mangelnden Pluralismus in der wirtschaftswissenschaftlichen Politikberatung, wodurch nicht nur die Qualität der Beratungsleistung leide, sondern auch die Entwicklung der ökonomischen Wissenschaft gehemmt würde. Sie schlagen vor, dass die Wirtschaftspolitik aktiv ein breiteres Spektrum an Beratungsleistungen nachfragen solle, das insbesondere solche Anbieter umfasst, die sich ausdrücklich eine ,heterodoxe“ Ausrichtung zu eigen machen.

Mit dem Vorwurf eines „fundamentalen Unverständnis der wissenschafts- und erkenntnistheoretischen Grundlagen“ an die Vertreter der ,unreformierten Orthodoxie" fahren die Autoren schweres Geschütz auf. Tatsächlich tritt aber diesbezüglich in den Abschnitten über die ,,erkenntnistheoretischen Grundlagen der Ökonomie als Politikberatende Wissenschaft“" und über die ,Wissenschaftlichkeit der wirtschaftspolitischen Beratung“"wenig zu Tage, was unter Ökonomen nicht seit langem breiter Konsens wäre. So maßen sich gerade die anwendungsorientierten, politikberatenden Ökonomen in der Regel ausdrücklich weder Entscheidungskompetenz noch überlegendes politisches Wissen an, sondern verstehen ihre Arbeit ganz im Sinne der völlig unstrittigen - instrumentellen Funktion, wie sie von den Autoren eingefordert wird. ${ }^{1}$ Dabei sollte jedem ernsthaften Wissenschaftler, ob Teil des Mainstreams oder nicht, die Vorläufigkeit allen Wissens im Popperschen Sinne klar sein. Eine „,objek-

\footnotetext{
${ }^{1}$ Hierauf haben beispielsweise die an der Gemeinschaftsdiagnose beteiligten Forschungsinstitute immer wieder hingewiesen. So heißt es in der Kapiteln „Zur Wirtschaftspolitik“ der jüngsten Gutachten: „die
}

This commentary article refers to the article available online at https://doi.org/10.1007/s41025-01900135-6. An author's reply to this commentary article is available online at https://doi.org/10.1007/ s41025-019-00149-0.

S. Kooths $(\bowtie)$

Prognosezentrum, Institut für Weltwirtschaft (IfW), Kiel, Deutschland

E-Mail: stefan.kooths@ifw-kiel.de

BiTS Business Leadership, University of Applied Sciences Europe (UE), Berlin, Deutschland 
tive Wahrheit" gefunden zu haben, wird niemand für sich in Anspruch nehmen. Es tut der Debatte nicht gut, die Gegenseite in die Nähe anderslautender Positionen zu rücken oder sie mit einem unterschwelligen Ideologieverdacht zu belegen.

Ein wissenschaftliches Paradigma zeichnet sich nicht durch Werturteile im Sinne politischer Ziele aus, sondern durch Festlegungen darüber, was im Bereich des jeweiligen Wissensgebietes für wesentlich gehalten wird und was nicht (Ratcliffe 1983, S. 165f). Paradigmen schaffen eine gewisse Ordnung und geben Orientierung, indem sie Wichtiges von Unwichtigem trennen. Durch die so erzielte Komplexitätsreduktion ermöglichen sie überhaupt erst das wissenschaftliche Arbeiten, weil der Mensch andernfalls von der Vielschichtigkeit der ihn umgebenden Welt überwältigt wäre. Paradigmen sind somit der beschränkten kognitiven Fähigkeit des Menschen geschuldet. Freilich kann der Mensch auch beim Aufstellen eines wissenschaftlichen Paradigmas irren, so dass diese offen bleiben müssen und immer nur vorläufige Gültigkeit beanspruchen können. Dies gilt uneingeschränkt auch für die ökonomische Wissenschaft, die sich mit komplexen Wirtschaftsprozessen befasst und diese theoretisch wie empirisch zu durchdringen sucht, nicht zuletzt, um auf dieser Grundlage wirtschaftspolitische Empfehlungen abzugeben.

Alternative Annahmen über die Funktionsweise des Wirtschaftsprozesses sind im analytischen Sinne normativ, sie stellen aber keine endgültigen Setzungen, sondern nur vorläufige Hypothesen dar, die sich wie alles andere in der Wissenschaft bewähren müssen. Auch paradigmatische Grundsatzfragen sind einer wissenschaftlichen Prüfung zugänglich, weil derjenige, der aus ideologischen Gründen an einer verzerrten Weltsicht festhält, letztlich „durch Misserfolge bestraft wird“ (Eucken 1954, S. 15). Das Trennen von Wesentlichem und Unwesentlichem zur Erklärung und Prognose des Wirtschaftsprozesses als Grundlage für wirtschaftspolitische Empfehlungen gehört somit untrennbar in die Sphäre der Wissenschaft. Die Politik als Adressat der wissenschaftlichen Beratung kann hier nicht den Schiedsrichter spielen, weil die Entscheidungskriterien wissenschaftlicher (erkenntnisgeleiteter) und nicht politischer (interessengeleiteter) Natur sind. Wahrheit ist insbesondere keine Frage der politischen Mehrheit. Die Förderung von heterodoxer Pluralität führt hier nicht weiter, sondern verlagert die Probleme nur auf eine andere Ebene. So müssten Politiker, denen sich widersprechende wirtschaftspolitische Empfehlungen aus der Wissenschaft vorgelegt werden, letztlich die Frage entscheiden, welcher Sicht sie folgen sollten oder welche analytischen Setzungen sie für eine ,ausgewogene Entscheidung" wie hoch gewichten und damit genau jene Aufgabe lösen, die die Wissenschaft noch nicht - zumindest nicht im Konsens - hat entscheiden können. Das überfordert klar die Rolle der politischen Entscheidungsträger, weil sich die Tragweite einzelner paradigmatischer Positionen kaum von Außenstehenden überblicken und durchdringen lässt. Im schlimmsten Fall käme es dazu, dass die wis-

Institute [wollen] auf brachliegende Wertschöpfungspotenziale hinweisen, die Wirtschaftspolitik auf Konsistenz und Nachhaltigkeit hin beurteilen und bei alternativen Maßnahmen diejenigen identifizieren, mit denen sich die politischen Ziele zu den geringsten ökonomischen Kosten erreichen lassen. " (Projektgruppe Gemeinschaftsdiagnose 2017, S. 61) und „Für die wirtschaftspolitische Beratung ist es wichtig, dass die Politik ihre Ziele klar benennt, denn nur dann lassen sich konkrete wirtschaftspolitische Maßnahmen ex-ante bewerten, um Handlungsempfehlungen abgeben zu können, und ex-post evaluieren, um für die Zukunft daraus zu lernen.“ (Projektgruppe Gemeinschaftsdiagnose 2018, S. 57). 
senschaftliche Expertise beliebig wird und sich jede politische Richtung durch ,die Wissenschaft" bestätigt fühlte. Damit gerieten Ökonomen in jene Ideologie- bzw. Alibifunktion, die ja gerade verhindert werden soll. Die Autoren suggerieren, dass die Wahrheit bei widerstreitenden Paradigmen irgendwo in der Mitte liegen könnte. Dafür gibt es erkenntnistheoretisch keinen Beleg. Wer auf eine Mauer zufährt, sich aber zwischen dem Ausweichen nach links oder rechts nicht entscheiden kann, ist schlecht beraten, wenn er als Kompromiss die vermeintlich goldene Mitte wählt. ${ }^{2}$

Darüber hinaus stellt sich die Frage, welche wirtschaftswissenschaftlichen Paradigmen durch entsprechende staatliche Nachfrageprogramme gefördert werden sollen. Ein everything goes würde den Erkenntnisfortschritt, den die ökonomische Wissenschaft in den letzten Jahrhunderten vorweisen kann, in Frage stellen. So besteht etwa kein Bedarf an wirtschaftspolitischer Beratung marxistischer Prägung, die auf dem Fundament einer überwundenen ricardianischen Arbeitswertlehre aufbaut. ${ }^{3}$ Die Frage, welche alternativen Richtungen im Sinne paradigmatischer Suchprozesse eingeschlagen werden, muss daher der Wissenschaft überlassen bleiben. Die Politik kann den Ökonomen diese Entscheidung nicht abnehmen, sie müssen diese anspruchsvolle Aufgabe schon selbst übernehmen.

Heterodoxe Pluralität als Nebeneinander verschiedener Denkschulen ist auch deshalb kein Ersatz für ein mühsames, nach den Kriterien der Wissenschaft geführtes Ringen um Erkenntnisfortschritt, weil das Einteilen der ökonomischen Wissenschaft in widerstreitende Schulen ein zu grobes Raster für die paradigmatische Weiterentwicklung darstellt. So führt die pauschale Kontroverse zwischen nachfrage- und angebotsorientierter Makroökonomik kaum weiter, sondern gefragt ist ein tiefer schürfendes kritisches Abklopfen der für die Analyse gesetzten Modellbedingungen im Sinne von ,„progressiven Problemverschiebungen“(Lakatos 1970). Dies sei kurz an wenigen Beispielen illustriert:

1. Kann die gesamtwirtschaftliche Produktion als zeitloses Phänomen aufgefasst werden, oder liegt gerade darin, dass sich Produktionsprozesse als Abfolge jeweils zeiterfordernder Stufen vollziehen, ein relevantes Merkmal für die Erklärung der gesamtwirtschaftlichen Aktivität?

\footnotetext{
2 Eine solche Situation zeigt sich seit längerem in der Diskussion um die Reform der Europäischen Währungsunion. Aufgrund der systemischen Interdependenz bringt es wenig, verschiedene wissenschaftlich fundierte Reformvorschläge in der Weise heranzuziehen, dass Elemente eines Hartwährungssystems (Geldwertstabilität, Nicht-Beistandsregel) mit denen eines Weichwährungssystems (monetäre Staatsfinanzierung, Vergemeinschaftung von Risiken) verbunden werden. Jedes System für sich könnte bessere Ergebnisse zeitigen als ein Mittelweg, der in Fragen der Währungsordnung zu einem „stuck-in-the-middle“ führte und eine kohärente Wirtschaftspolitik verhinderte.

3 Dies bedeutet ausdrücklich nicht, dass sich die Hochschullehre nicht mit marxistischer Theorie auseinanderzusetzen hätte - im Gegenteil. Ein Ökonom sollte durch ein entsprechendes theoretisches Verständnis in der Lage sein, sich selbst ein fundiertes Bild dieses Theoriestrangs zu machen und sich nicht nur darauf verlassen müssen, dass andere es widerlegt hätten oder sich gar nur mit dem empirischen Befund der gescheiterten sozialistischen Zentralveraltungswirtschaften zufriedengeben. Nicht zuletzt führt die kritische Auseinandersetzung mit marxistischer Theorie und ihren Fehlern auch zu einem erheblich besseren Verständnis im Gesamtbereich der Wert-, Kapital- und Zinstheorien, wie es Böhm-Bawerk (1896) eindrucksvoll unter Beweis gestellt hat.
} 
2. Lässt sich die Schwankung der Wirtschaftsleistung primär durch eine Niveaubetrachtung großer Aggregate oder vielmehr durch deren Zusammensetzung (Strukturdiskrepanzen) erklären?

3. Genügt es, für Potenzialbetrachtungen (und damit für die Bestimmung der gesamtwirtschaftlichen Auslastungsgrades) den Kapitalstock als homogenen Fonds zu betrachten, oder kommt es darauf an, die heterogene Kapitalgüterstruktur, die eine Vorfestlegung der damit möglichen Produktion impliziert, in den Mittelpunkt der Analyse zu rücken?

4. Kann die monetäre Analyse auf Preisniveauwirkungen beschränkt werden, oder spielen Preisstrukturwirkungen und Cantillon-Effekte eine wichtige Rolle zur Beurteilung der Geldpolitik?

5. Lässt sich der Wirtschaftsprozess als Abfolge von Gleichgewichten darstellen, oder ist es zweckmäßiger, von einer Ungleichgewichtsdynamik mit FeedbackMechanismen auszugehen?

6. Kann in der Erklärung marktwirtschaftlicher Prozesse von der Rolle des unternehmerischen Handelns abstrahiert werden, oder muss die ökonomische Erklärung gerade beim Verhalten unternehmerischer Akteure ihren Ausgang nehmen?

Entlang dieser und ähnlich gelagerter Fragen über den Erkenntnisgegenstand sollte sich eine ernsthafte paradigmatische Debatte in der ökonomischen Wissenschaft bewegen. In dem Maße, wie sich dadurch die Prognosefähigkeit der Theorie erhöht, verbessert sich auch die wirtschaftspolitische Beratung. Eucken (1954, S. $20 \mathrm{ff})$ hat mit den drei modelltheoretischen Bedingungsfehlern (unmögliche, unnötige und einseitige Bedingungen) die wesentlichen Kriterien umrissen, die für eine solche evolutionäre Fortentwicklung der ökonomischen Wissenschaft weiterhin gültig sind. ${ }^{4}$

Das Einschätzen der theoretischen Implikationen wie auch das Beurteilen der empirischen Relevanz dieser jeweiligen Weichenstellungen zählen zu den wissenschaftlich anspruchsvollsten Aufgaben der Ökonomen, die sich hierfür neben einem breiten Methodenwissen auch vertiefte Kenntnisse über die in der Vergangenheit bereits verfolgten Ansätze aneignen müssen (theoriegeschichtliches Fundament). Welche paradigmatischen Wege verschiedene Wissenschaftler dann vor diesem Hintergrund einschlagen, muss den Wissenschaftlern selbst überlassen bleiben - genau dafür sind sie Wissenschaftler. In dem Maße, wie die für ein eigenes Urteil erforderliche theoriegeschichtliche Ausbildung im akademischen Bereich zu kurz kommt, droht in der Tat eine Verengung im Spektrum. Insbesondere droht bei der Dominanz einer bestimmten Methodik (etwa einer Überbetonung der Mathematik als Sprache der Ökonomen) der Fehlschluss, dass die Mainstream-Forschungsgrenze bereits al-

\footnotetext{
4 Man kann - ausgehend von einem harten Kern des ökonomischen Wissens - diesen Suchprozess auch als eine Variante der Popperschen „Stückwerktechnik“ (piecemeal engineering) auffassen (Popper 1957), die nun aber nicht auf das Institutionendesign, sondern auf den Theoriefortschritt selbst bezogen ist. In ähnliche Richtung argumentiert Lakatos (1968), wenn er den ursprünglichen Popperschen Falsifikationismus (Popper 1934) zu einem „raffinierten Falsifikationismus“ (sophisticated methodological falsificationism) weiterentwickelt, wobei er davon ausgeht, dass die evolutionär vorangetriebene Weiterentwicklung eines Forschungsprogramms der Überprüfung auf Übereinstimmung mit den beobachtbaren Tatsachen prinzipiell zugänglich ist.
} 
les beinhaltet, was jemals an richtigen Erkenntnissen erdacht worden ist. Weil sich mit einer bestimmten Sprache nicht alles gleichermaßen ausdrücken lässt, kann auf diese Weise auch wichtiges Wissen wieder verloren gehen - hierzu finden sich prominente Beispiele für alle unter (1) bis (6) angeführten paradigmatischen Bausteine. Nur durch die Beschäftigung mit der Theoriegeschichte (und ihren zahlreichen Irrwegen und Sackgassen) kann ein Wissenschaftler tatsächlich auf die Schultern der akademischen Vorfahren hinaufklettern und zu einem abgewogeneren Urteil über den State-of-the-art gelangen. Wer nur den derzeitigen Mainstream kennt, steht indessen nicht automatisch auf den Schultern der Ahnen, weil dieser immer nur einen Ausschnitt des ökonomischen Wissensgebäudes abbilden kann (Garrison 1993). Es gibt daher gute Gründe, den theoriegeschichtlichen Bereich nicht geringzuschätzen. Eventuelle Defizite können aber nur im wissenschaftlichen Diskurs selbst adressiert und nicht durch staatliche Stellen vorgeschrieben werden, solange die Wissenschaft ihre akademische Freiheit wahren will.

Der Wirtschaftspolitik wie auch der Öffentlichkeit wäre wenig gedient, wenn die wirtschaftswissenschaftlichen Beratungsinstanzen wie die Sachverständigenräte oder die Gemeinschaftsdiagnose ihre Aufgabe in erster Linie darin sähen, ihre Uneinigkeit vor den Adressaten auszubreiten. Informativ im Sinne eines wissenschaftlich fundierten Inputs für wirtschaftspolitische Entscheidungen dürfte vielmehr sein, herauszuarbeiten, worauf sich die beteiligten Wissenschaftler - bei allen individuellen Unterschieden - letztlich einigen können. Von daher hat das, worauf sie sich im Konsens als wissenschaftlich fundiert verständigen können, ein höheres Gewicht als die Streitfälle. Dass dabei die Empfehlungen zuweilen weniger prägnant ausfallen, liegt in der Natur des Kompromisses. Diesen sollten die beteiligten Wissenschaftler aber besser abwägen können als die Adressaten ihrer Empfehlungen. Zwar müssen Minderheitsvoten zulässig sein. Es ist aber nicht zu kritisieren, dass diese die Ausnahme bleiben sollten und die Mitglieder von Beratungsgremien aufgerufen sind, ein gemeinsames Votum anzustreben. So wären fünf Einzelgutachten der Mitglieder des Sachverständigenrates zur Begutachtung der gesamtwirtschaftlichen Entwicklung mit Blick auf den Stand der Wissenschaft weniger informativ als ein Gemeinschaftsgutachten.

Es spricht vieles dafür, die Mitgliedschaft in wissenschaftlichen Beiräten zeitlich zu begrenzen, allerdings sollten die jeweiligen Mandate deutlich über eine Legislaturperiode hinausgehen und gerade nicht mit diesen synchronisiert werden. Die von den Autoren vorgeschlagene vollständige Neuberufung zu Beginn einer Legislaturperiode, um Wertkongruenz mit der jeweiligen Regierungsmehrheit herzustellen, ist aus zweierlei Gründen nicht zu empfehlen. In der Pluralismusdebatte sollte es gerade nicht um politische Ziele der wissenschaftlichen Berater gehen (Werturteile), sondern um paradigmatische Diversität (Methoden- und Theorievielfalt). Dieser Vorschlag führt völlig unnötig zu einer Politisierung der wirtschaftswissenschaftlichen Kontroversen. Darüber hinaus wäre für die Pluralität wenig gewonnen, wenn mit einem Regierungswechsel die Beratungsgremien ausgetauscht würden und es so letztlich zu einer Kaskade von Monokulturen käme.

In dem Maße, wie die Autoren vor allem paradigmatische Transparenz einfordern wollen, ist ihnen uneingeschränkt zuzustimmen. Die Auseinandersetzung darüber kommt tatsächlich zu kurz, mitunter wird der Begriff des Paradigmas selbst 
nur mit spitzen Fingern angefasst, um nicht Gefahr zu laufen, als vorurteilsbehaftet und damit als unwissenschaftlich zu gelten. Dabei ist es für einen Wissenschaftler unmöglich, keinem Paradigma zu folgen (Kuhn 1970, S. 109). Es kann allenfalls nur implizit vorliegen, sofern es nicht bewusst reflektiert wird, was aus wissenschaftlicher Sicht tatsächlich ein schwerwiegendes Versäumnis wäre. Wissenschaft setzt intersubjektive Nachvollziehbarkeit voraus, daher ist es unabdingbar, dass Wissenschaftler ihr jeweiliges Paradigma offenlegen und sorgfältig dokumentieren. Dies dient nicht nur der Abgrenzung, sondern auch der internen Kohärenz. Wie divers sich dann die Wissenschaftslandschaft ausprägt, kann aber immer nur das endogene Ergebnis wissenschaftsinterner Prozesse sein und nicht von außen vorgegeben werden. Es ist daher zu begrüßen, dass die wirtschaftspolitischen Instanzen in Deutschland etwa im Rahmen der Gemeinschaftsdiagnose ausdrücklich an methodischer Pluralität interessiert sind, den Anbietern diesbezüglich aber keine inhaltlichen Vorgaben machen. ${ }^{5}$

Niemand bestreitet, dass der wissenschaftliche Fortschritt auf Pluralität (im Sinne von Methodenvielfalt und Ergebnisoffenheit) angewiesen ist und dass die entscheidenden Durchbrüche zu neuer Erkenntnis typischerweise zunächst aus einer Minderheitenposition heraus vertreten werden mussten. Der paradigmatische Wandel ist in der Tat ein nur langsam fortschreitender Prozess, der bekanntermaßen bereits von Max Planck beklagt wurde. ${ }^{6}$ Das mag man bedauern, aber auch hier ist der Staat nicht der geeignete Impulsgeber, der der Wissenschaft durch seine Auftragsvergabe auf die Sprünge helfen kann - im Gegenteil. Da staatliche Stellen nur solche Paradigmen nachfragen können, die bereits bekannt sind, würde dies den wissenschaftlichen Fortschritt tendenziell verlangsamen als beschleunigen.

Das Bild, das die Autoren zur Relevanz und Übertragbarkeit theoretischer Forschungsergebnisse in wirtschaftspolitisches Handeln zeichnen, ist schwer nachvollziehbar. Zum einen haben Beratungsinstanzen, die zu wirtschaftspolitisch relevanten Fragen arbeiten, einen klaren Wettbewerbsvorteil in der operativen Politikberatung. Zum anderen scheinen die Autoren mit den Ergebnissen einer breiten Literatur, die die begrenzten Erfolgsaussichten der Wirtschaftspolitik mit Blick auf die Planbarkeit ökonomischer Ergebnisse zum Gegenstand hat, zu hadern (grundlegend zur Interventionismusdebatte Mises 1998 und Hayek 2009). Dies liegt aber nicht an einer ,ideologisch motivierten Interpretation“ von theoretischen Modellergebnissen, sondern liegt in der Komplexität des ökonomischen Systems selbst begründet, das aufgrund seiner Eigenschaften nach allen bislang bekannten Koordinationsmechanismen der wirtschaftspolitischen Feinsteuerung Grenzen setzt. Gerade Mises und Hayek haben ihrer Forschung gerade kein neoklassisches Standardmodell zugrunde gelegt, sondern ihre Ergebnisse ausgehend von einer lebensnahen Charakterisierung

\footnotetext{
5 So hieß es in der Ausschreibung der Bundesregierung zur Gemeinschaftsdiagnose (GD) für den Vergabezeitraum 2016-2020: „Die GD ist ein gemeinsames Forschungsprojekt mehrerer Wirtschaftsforschungsinstitute. Durch ihre Zusammenarbeit werden die Analyse und die Prognose im Dialog und im Wettstreit mit verschiedenen theoretischen und methodischen Ansätzen bestmöglich fundiert."

6 ,Eine neue wissenschaftliche Wahrheit pflegt sich nicht in der Weise durchzusetzen, daß ihre Gegner überzeugt werden und sich als belehrt erklären, sondern vielmehr dadurch, daß ihre Gegner allmählich aussterben und daß die heranwachsende Generation von vornherein mit der Wahrheit vertraut gemacht ist." (Planck 1948, S. 22).
} 
der ökonomischen Akteure abgeleitet (homo agens statt homo oeconomicus). ${ }^{7} \mathrm{Zu}-$ dem bemüht sich die empirisch-evidenzbasierte Politikberatung ausdrücklich um eine Erfolgskontrolle wirtschaftspolitischer Eingriffe. Es ist daher unverständlich, wie die Autoren zu der Einschätzung gelangen, es mangele an einem „Bewusstsein [...], theoretische und empirische Erkenntnisse der Ökonomie aus einer wirtschaftspolitischen Sicht wissenschaftlich zu hinterfragen“.

Schließlich hätte es dem Argument für mehr Pluralität in der wirtschaftswissenschaftlichen Beratungslandschaft gutgetan, wenn die Autoren durch die Wahl ihrer Beispiele nicht nur auf keynesianische Erklärungsalternativen hingewiesen hätten. So entsteht der Eindruck, ihnen ginge es in erster Linie darum, ihre Sicht der Dinge $\mathrm{zu}$ rechtfertigen und weniger der Pluralität als solche das Wort zu reden. So haben die Vertreter der Austrian Economics allen Grund, sich durch die Weltfinanzkrise 2007/2008 in ihrem Paradigma bestätigt zu fühlen (Huerta de Soto 2009; Tempelmann 2010), wobei sie diese zudem in den größeren Kontext einer Krise des Makro-Managements einordnen können und dabei aufzeigen, wie das MainstreamParadigma sinnvoll erweitert werden könnte (Kirzner 1966, Horwitz 2000, Garrison 2001). Anders als die Autoren suggerieren, ist ihre keynesianische Position (aktive Konjunkturpolitik als Reaktion auf den Produktionseinbruch 2008/2009) nicht im Nachhinein durch die Daten bestätigt oder gar als Konsens in den Mainstream eingegangen. Während der Ausbau der automatischen Stabilisatoren (Kurzarbeiterregelung) die Widerstandsfähigkeit der deutschen Wirtschaft gestärkt haben dürfte, wirkten die Konjunkturprogramme im Wesentlichen erst, als die Krise schon wieder überwunden war. Zudem konnte der Staat die schlagartig wegfallende Export- und Investitionsgüternachfrage ohnehin nicht kompensieren. Hier ist die keynesianische Erklärung weiterhin blind dafür, dass mit den gegebenen Produktionsstrukturen nicht jeder beliebige Verwendungsvektor bedient werden kann. Mithin hätte auch ein frühzeitigeres Einschwenken auf keynesianische Rezepte oder eine stärkere Gewichtung keynesianischer Empfehlungen die Krise kaum entschärfen können.

Fazit: Die Forderung nach paradigmatischer Offenheit und Methodenvielfalt ist berechtigt, allerdings auch nicht kontrovers. Der Vorschlag, den wissenschaftlichen Erkenntnisprozess diesbezüglich zu fördern, indem der Staat als Nachfrager für heterodoxe Politikberatung auftritt, kann indes nicht überzeugen. Dieser End-of-pipeAnsatz setzt voraus, dass staatliche Stellen das richtige Maß an Pluralität besser einschätzen können als die wissenschaftliche Gemeinschaft selbst. Genau das zeigt der Beitrag von Sebastian Dullien und Gustav Horn aber nicht. Stattdessen erschwert er die zu Recht eingeforderte paradigmatische Klarheit in der wirtschaftspolitischen Beratung, indem wissenschaftlich entscheidbare Fragen mit der Werturteilsproblematik in den Sozialwissenschaften vermengt werden.

\footnotetext{
7 Auch die von den Autoren behauptete Asymmetrie zur Verteidigung des Status-quo ist zweifelhaft. So wird die neoklassische Modellwelt nicht selten auch dazu herangezogen, eine Idealwelt zu formulieren, an der die Realität aufgrund tatsächlicher oder vermeintlicher Marktversagenstatbestände nur scheitern kann, woraus dann unter der Fiktion eines zentralen sozialen Planers Interventionsspielräume abgeleitet werden. Eine solche Analyse lässt aber nicht nur die Rolle unternehmerischer Akteure als universelle Arbitrageure und Innovatoren außen vor (etwa zur Lösung des vermeintlichen Market-for-Lemons-Problems), sondern überschätzt zugleich die Informationsbasis der tatsächlich handelnden staatlichen Akteure.
} 


\section{Literatur}

v. Böhm-Bawerk, E. (1896). Zum Abschluß des Marxschen Systems. In O. v. Boenigk (Hrsg.), Staatswissenschaftliche Arbeiten. Festgaben für Karl Knies zur fünfundsiebzigsten Wiederkehr seines Geburtstages in dankbaren Verehrung (S. 87-205). Berlin: O. Haering.

Eucken, W. (1954). Kapitaltheoretische Untersuchungen (2. Aufl.). Tübingen: J. C. B. Mohr (Paul Siebeck).

Garrison, R. W. (1993). Mises and his methods. In J. M. Herbener (Hrsg.), The meaning of Ludwig von Mises - contributions in economics, epistemology, sociology, and political philosophy. Studies in Austrian Economics Series. Norwell: Kluwer.

Garrison, R.W. (2001). Time and money - the macroeconomics of capital structure. Foundations of the market economy. London, New York: Routledge.

Hayek, F. A. (2009). Collectivist economic planning. Auburn: Ludwig von Mises Institute.

Horwitz, S. (2000). Microfoundations and macroeconomics - an Austrian perspective. Foundations of the market economy. London, New York: Routledge.

Huerta de Soto, J. (2009). Money, bank credit, and economic cycles (3. Aufl.). Auburn: Ludwig von Mises Institute.

Kirzner, I. M. (1966). An essay on capital. New York: Augustus M. Kelley.

Kuhn, T.S. (1970). The structure of scientific revolutions (2. Aufl.). International encyclopedia of unified science, Bd. Volume II, Number 2. Chicago: University of Chicago Press.

Lakatos, I. (1968). Criticism and the methodology of scientific research programmes. Proceedings of the Aristotelian Society, 69(1), 149-186.

Lakatos, I. (1970). Falsification and the methodology of scientific research programmes. In I. Lakatos \& A. Musgrave (Hrsg.), Criticism and the growth of knowledge (S. 91-196). Cambridge: Cambridge University Press.

v. Mises, L. (1998). Interventionism - an economic analysis. New York: Foundation for Economic Education.

Planck, M. (1948). Wissenschaftliche Selbstbiographie. Leipzig: Johann Ambrosius Barth.

Popper, K. (1934). Logik der Forschung - Zur Erkenntnistheorie der modernen Naturwissenschaft. Wien: Springer.

Popper, K. (1957). The poverty of Hirstoricism. London: Routledge.

Projektgruppe Gemeinschaftsdiagnose (2017). Aufschwung weiter kräftig - Anspannungen nehmen zu. Gemeinschaftsdiagnose im Herbst 2017, Kiel. Berlin: Deutsches Institut für Wirtschaftsforschung (DIW).

Projektgruppe Gemeinschaftsdiagnose (2018). Deutsche Wirtschaft im Boom - Luft wird dünner. Gemeinschaftsdiagnose im Frühjahr 2018, München. Berlin: Deutsches Institut für Wirtschaftsforschung (DIW).

Ratcliffe, J. W. (1983). Notions of validity in qualitative research methodology. Knowledge. Creation, Diffusion, Utilization, 5(2), 147-167.

Tempelmann, J.H. (2010). Austrian business cycle theory and the global financial crisis: Confessions of a mainstream economist. Quarterly Journal of Austrian Economics, 13(1), 3-15. 